\title{
Impact of neurosurgeon specialization on patient outcomes for intracranial and spinal surgery: a retrospective analysis of the Nationwide Inpatient Sample 1998-2009
}

\author{
*Brandon A. McCutcheon, MD, MPP, 1,2 Brian R. Hirshman, MD, MS, ${ }^{1}$ Brandon C. Gabel, MD, ${ }^{1}$ \\ Michael W. Heffner, BA, ${ }^{1}$ Logan P. Marcus, MD, MS, ${ }^{1}$ Tyler S. Cole, MD, ${ }^{3}$ Clark C. Chen, MD, PhD, ${ }^{1}$ \\ David C. Chang, PhD, MBA, MPH, ${ }^{1,4}$ and Bob S. Carter, MD, PhD ${ }^{1}$ \\ 1Department of Neurosurgery, University of California, San Diego, La Jolla, California; 2Department of Neurologic Surgery, Mayo \\ Clinic and Mayo Clinic Foundation, Rochester, Minnesota; ${ }^{3}$ Barrow Neurological Institute, Phoenix, Arizona; and ${ }^{4}$ Department of \\ Surgery, Massachusetts General Hospital, Boston, Massachusetts
}

\begin{abstract}
OBJECTIVE The subspecialization of neurosurgical practice is an ongoing trend in modern neurosurgery. However, it remains unclear whether the degree of surgeon specialization is associated with improved patient outcomes. The authors hypothesized that a trend toward increased neurosurgeon specialization was associated with improved patient morbidity and mortality rates.
\end{abstract}

METHODS The Nationwide Inpatient Sample (NIS) was used (1998-2009). Patients were included in a spinal analysis cohort for instrumented spine surgery involving the cervical spine (International Classification of Diseases, Ninth Revision, Clinical Modification [ICD-9-CM] codes 81.31-81.33, 81.01-81.03, 84.61-84.62, and 84.66) or lumbar spine (codes 81.04-81.08, 81.34-81.38, 84.64-84.65, and 84.68). A cranial analysis cohort consisted of patients receiving a parenchymal excision or lobectomy operation (codes 01.53 and 01.59). Surgeon specialization was measured using unique surgeon identifiers in the NIS and defined as the proportion of a surgeon's total practice dedicated to cranial or spinal cases.

RESULTS A total of 46,029 and 231,875 patients were identified in the cranial and spinal analysis cohorts, respectively. On multivariate analysis in the cranial analysis cohort (after controlling for overall surgeon volume, patient demographic data/comorbidities, hospital characteristics, and admitting source), each percentage-point increase in a surgeon's cranial specialization (that is, the proportion of cranial cases) was associated with a 0.0060 reduction in the log odds of patient mortality (95\% Cl $0.0034-0.0086)$ and a 0.0042 reduction in the log odds of morbidity $(95 \% \mathrm{Cl} 0.0032-0.0052)$. This resulted in a $15 \%$ difference in the predicted probability of mortality for neurosurgeons at the 75th versus the 25th percentile of cranial specialization. In the spinal analysis cohort, each percentage-point increase in a surgeon's spinal specialization was associated with a 0.0122 reduction in the log odds of mortality $(95 \% \mathrm{Cl} 0.0074-0.0170)$ and a 0.0058 reduction in the log odds of morbidity $(95 \% \mathrm{Cl} 0.0049-0.0067)$. This resulted in a $26.8 \%$ difference in the predicted probability of mortality for neurosurgeons at the 75th versus the 25th percentile of spinal specialization.

CONCLUSIONS For both spinal and cranial surgery patient cohorts derived from the NIS database, increased surgeon specialization was significantly and independently associated with improved mortality and morbidity rates, even after controlling for overall case volume.

https://thejns.org/doi/abs/10.3171/2016.4.JNS152332

KEY WORDS specialization; NIS; multivariate analysis; surgical outcomes research; spinal fusion; lobectomy; parenchymal excision; Nationwide Inpatient Sample

ABBREVIATIONS ICD-9-CM = International Classification of Diseases, Ninth Revision, Clinical Modification; IQR = interquartile range; NIS = Nationwide Inpatient Sample. SUBMITTED October 6, 2015. ACCEPTED April 7, 2016.

* Drs. McCutcheon and Hirshman contributed equally to this work. 
$\mathrm{O}$ VER the past half century, there has been a tendency toward subspecialization. By the 1970s, there were more than 20 recognized medical and surgical subspecialties with their own board-certification processes. ${ }^{4}$ As a result, surgical subspecialization is becoming the norm throughout the health care community. ${ }^{6}, 15$

Similar trends have been observed within neurosurgery. Although graduates of accredited neurosurgical programs are licensed to practice the gamut of neurosurgery, few exercise this prerogative. For example, hospitals routinely require fellowship training to perform pediatric operations. ${ }^{11,18}$ Many neurosurgeons, moreover, gravitate toward a particular subspecialty out of choice. The reasons behind this are myriad but include the operating surgeon's natural inclinations, fellowship training, technical abilities, and/ or research interests. Academic departments may also encourage faculty members to subspecialize.

Data from multiple surgical specialties seem to support surgeon specialization. For example, Golledge et al. found that surgical subspecialization was associated with improved outcomes in patients with breast cancer. ${ }^{13} \mathrm{Com}-$ pared with general surgeons, vascular surgeons have lower mortality rates among patients when operating on abdominal aortic aneurysms..$^{10}$ Other work has demonstrated that patients of cardiothoracic surgeons have better outcomes after undergoing lung resection compared with those of general surgeons. ${ }^{14}$ Data on neurosurgical subspecialization are less robust, but some authors have shown that gross-total resection of intracranial neoplasms is higher in pediatric patients if the surgeon is subspecialized in pediatrics. $^{2,19}$

This study uses a national data set to investigate the impact of neurosurgical subspecialization on patient outcomes for both cranial and spinal surgery. We hypothesized that a trend toward increased neurosurgeon specialization was associated with improved patient outcomes.

\section{Methods \\ Data Source}

The Nationwide Inpatient Sample (NIS) from 1998 to 2009 was used as the data source for this study. These data are maintained by the Healthcare Cost and Utilization Project of the Agency for Healthcare Research and Quality. The NIS is the largest national administrative database for all payer categories and captures 100\% of discharges from a $20 \%$ stratified sample of US hospitals. Because the NIS uses a stratified randomized sampling technique, the data contained in the NIS are representative of and generalizable to US hospitals. Within the NIS, the unit of analysis is a patient admission. Each admission record includes International Classification of Diseases, Ninth Revision, Clinical Modification (ICD-9-CM) codes for the 15 most important procedures performed during that admission, as well as a unique identifier enabling identification of the primary provider.

\section{Identification of Neurosurgeons and Surgeon Operative Volume}

A provider was considered for inclusion in the study if he or she performed any of the cranial or spinal operations listed in Table 1. Providers were linked across multiple patient admissions using a unique identifier as done in similar studies using the NIS, 3,7,12,16 Total operative volume for a provider-year was equal to the total number of cranial and spinal operations performed in a calendar year. Because the provider identifiers do not correlate across calendar years, ${ }^{12}$ our unit of analysis for this work was the neurosurgeon-year, defined as the set of all patients who underwent operations by a neurosurgeon during a calendar year.

To ensure that our data set contained only neurosurgeons, 2 exclusion criteria were applied. First, because spine operations were also performed by orthopedic surgeons, surgeons were excluded from analysis if they did not perform at least 1 cranial operation as defined in Table 1. Second, to ensure that providers were actually active staff at their hospitals, neurosurgeons were excluded if they were not associated with a total volume of at least 50 cases in a given year.

\section{Neurosurgeon Specialization}

Neurosurgeon spinal specialization was computed for each neurosurgeon-year by determining the percentage of spinal operations (Table 1) of the total cranial and spinal operations performed by each provider in a given year. Cranial specialization was computed for each neurosurgeon-year by determining the percentage of cranial operations of the total cranial and spinal volume in a given year. For discussion in the present study, when a surgeon devoted more than $75 \%$ of their practice to either cranial or spinal surgery, they are described as highly specialized in either cranial or spinal surgery.

\section{Analysis Cohorts}

To ensure a viable comparison between the types of procedures done by surgeons of varying degrees of specialization, we chose a set of cranial and spinal codes that 1) had a degree of technical difficulty such that mortality differences might be apparent, and 2) were highly represented across the range of specialization. First, a cranial analysis cohort included all patients who underwent a cranial operation involving parenchymal excision or lobectomy (ICD-9-CM codes 01.53 and 01.59); these are codes that are typically used for mass lesion excision in the brain. A second spinal analysis cohort included all patients who underwent any of the following instrumented spinal operations: primary fusion of the cervical (codes 81.01-81.03) or lumbar (codes 81.04-81.08) spine, refusion of the cervical (codes 81.31-81.33) or lumbar (codes 81.34-81.38) spine, primary insertion of disc prosthesis of the cervical (codes 84.61-84.62) or lumbar (codes 84.64-84.65) spine, or revision of a disc prosthesis in the cervical (code 84.66) or lumbar (code 84.68) spine.

\section{Patient Outcomes}

Two primary end points were assessed during this study. The first end point was inpatient mortality, as recorded in the NIS. The second end point, adverse discharge disposition, has been used in previous neurosurgical studies within the NIS ${ }^{8}$ and was defined as discharge 
TABLE 1. Cranial and spine operation codes

\begin{tabular}{|c|c|}
\hline Code & Description \\
\hline \multicolumn{2}{|l|}{ Cranial } \\
\hline 01.01 & Cisternal puncture \\
\hline 01.02 & Ventriculopuncture through previously implanted catheter \\
\hline 01.09 & Other cranial puncture \\
\hline 01.10 & Intracranial pressure monitoring \\
\hline 01.11 & Closed percutaneous needle biopsy of cerebral meninges \\
\hline 01.12 & Open biopsy of cerebral meninges \\
\hline 01.13 & Closed percutaneous needle biopsy of brain \\
\hline 01.14 & Open biopsy of brain \\
\hline 01.15 & Biopsy of skull \\
\hline 01.16 & Intracranial oxygen monitoring \\
\hline 01.21 & Incision \& drainage of cranial sinus \\
\hline 01.22 & Removal of intracranial neurostimulator lead(s) \\
\hline 01.24 & Other craniotomy \\
\hline 01.25 & Other craniectomy \\
\hline 01.26 & Insertion of catheter(s) into cranial cavity or tissue \\
\hline 01.28 & Placement of intracerebral catheter(s) via bur hole(s) \\
\hline 01.31 & Incision of cerebral meninges \\
\hline 01.32 & Lobotomy \& tractotomy \\
\hline 01.39 & Other incision of brain \\
\hline 01.41 & Ops on thalamus \\
\hline 01.42 & Ops on globus pallidus \\
\hline 01.51 & Excision of lesion or tissue of cerebral meninges \\
\hline 01.52 & Hemispherectomy \\
\hline 01.53 & Lobectomy of brain \\
\hline 01.59 & Other excision or destruction of lesion or tissue of brain \\
\hline 02.11 & Simple suture of dura mater of brain \\
\hline 02.12 & Other repair of cerebral meninges \\
\hline 02.13 & Ligation of meningeal vessel \\
\hline 02.14 & Choroid plexectomy \\
\hline 02.31 & Ventricular shunt to structure in head \& neck \\
\hline 02.32 & Ventricular shunt to circulatory system \\
\hline 02.33 & Ventricular shunt to thoracic cavity \\
\hline 02.34 & Ventricular shunt to abdominal cavity \& organs \\
\hline 02.39 & Other ops to establish drainage of ventricle \\
\hline 02.41 & Irrigation \& exploration of ventricular shunt \\
\hline 02.42 & Replacement of ventricular shunt \\
\hline 02.43 & Removal of ventricular shunt \\
\hline 02.92 & Repair of brain \\
\hline 02.93 & $\begin{array}{l}\text { Implantation or replacement of intracranial neurostimulator } \\
\text { lead(s) }\end{array}$ \\
\hline 02.96 & Insertion of sphenoidal electrodes \\
\hline \multicolumn{2}{|l|}{ Spine } \\
\hline 03.01 & Removal of foreign body from spinal canal \\
\hline 03.02 & Reopening of laminectomy site \\
\hline 03.09 & Other exploration \& decompression of spinal canal \\
\hline 03.21 & Percutaneous chordotomy \\
\hline 03.29 & Other chordotomy \\
\hline 03.32 & Biopsy of spinal cord or spinal meninges \\
\hline 03.51 & Repair of spinal meningocele \\
\hline
\end{tabular}

» CONTINUED FROM PREVIOUS COLUMN

\section{TABLE 1. Cranial and spine operation codes}

\begin{tabular}{|c|c|}
\hline Code & Description \\
\hline \multicolumn{2}{|c|}{ Spine (continued) } \\
\hline 03.52 & Repair of spinal myelomeningocele \\
\hline 03.53 & Repair of vertebral fracture \\
\hline 03.59 & Other repair \& plastic operations on spinal cord structures \\
\hline 03.71 & Spinal subarachnoid-peritoneal shunt \\
\hline 03.72 & Spinal subarachnoid-ureteral shunt \\
\hline 03.79 & Other shunt of spinal theca \\
\hline 03.93 & $\begin{array}{l}\text { Implantation or replacement of spinal neurostimulator } \\
\text { lead(s) }\end{array}$ \\
\hline 03.94 & Removal of spinal neurostimulator lead(s) \\
\hline 03.97 & Revision of spinal thecal shunt \\
\hline 03.98 & Removal of spinal thecal shunt \\
\hline 80.50 & Excision or destruction of intervertebral disc, unspecified \\
\hline 80.51 & Excision of intervertebral disc \\
\hline 80.52 & Intervertebral chemonucleolysis \\
\hline 80.53 & Repair of the anulus fibrosus $w /$ graft or prosthesis \\
\hline 80.54 & Other \& unspecified repair of the anulus fibrosus \\
\hline 80.59 & Other destruction of intervertebral disc \\
\hline 81.00 & Spinal fusion, not otherwise specified \\
\hline 81.01 & Atlas-axis spinal fusion \\
\hline 81.02 & Other cervical fusion, anterior technique \\
\hline 81.03 & Other cervical fusion, posterior technique \\
\hline 81.04 & Dorsal \& dorsolumbar fusion, anterior technique \\
\hline 81.05 & Dorsal \& dorsolumbar fusion, posterior technique \\
\hline 81.06 & Lumbar \& lumbosacral fusion, anterior technique \\
\hline 81.07 & $\begin{array}{l}\text { Lumbar \& lumbosacral fusion, lateral transverse process } \\
\text { technique }\end{array}$ \\
\hline 81.08 & Lumbar \& lumbosacral fusion, posterior technique \\
\hline 81.30 & Refusion of spine, not otherwise specified \\
\hline 81.31 & Refusion of atlas-axis spine \\
\hline 81.32 & Refusion of other cervical spine, anterior technique \\
\hline 81.33 & Refusion of other cervical spine, posterior technique \\
\hline 81.34 & Refusion of dorsal \& dorsolumbar spine, anterior technique \\
\hline 81.35 & Refusion of dorsal \& dorsolumbar spine, posterior technique \\
\hline 81.36 & Refusion of lumbar \& lumbosacral spine, anterior technique \\
\hline 81.37 & $\begin{array}{l}\text { Refusion of lumbar \& lumbosacral spine, lateral transverse } \\
\text { process technique }\end{array}$ \\
\hline 81.38 & $\begin{array}{l}\text { Refusion of lumbar \& lumbosacral spine, posterior tech- } \\
\text { nique }\end{array}$ \\
\hline 81.39 & Refusion of spine, not elsewhere classified \\
\hline 81.62 & Fusion or refusion of 2-3 vertebrae \\
\hline 81.63 & Fusion or refusion of $4-8$ vertebrae \\
\hline 81.64 & Fusion or refusion of 9 or more vertebrae \\
\hline 84.51 & Insertion of interbody spinal fusion device \\
\hline 84.59 & Insertion of other spinal devices \\
\hline 84.60 & Insertion of spinal disc prosthesis, not otherwise specified \\
\hline 84.65 & Insertion of total spinal disc prosthesis, lumbosacral \\
\hline 84.66 & $\begin{array}{l}\text { Revision or replacement of artificial spinal disc prosthesis, } \\
\text { cervical }\end{array}$ \\
\hline
\end{tabular}

Spine (continued)

03.52 Repair of spinal myelomeningocele

03.53 Repair of vertebral fracture

03.59 Other repair \& plastic operations on spinal cord structures

03.71 Spinal subarachnoid-peritoneal shunt

03.72 Spinal subarachnoid-ureteral shunt

03.79 Other shunt of spinal theca

03.94 Removal of spinal neurostimulator lead(s)

3.97 Revision of spinal thecal shunt

98 Removal of spinal thecal shun

0.50 Excision or destruction of intervertebral disc, unspecified

51 Excision of intervertebral disc

0.52 Intervertebral chemonucleolysis

80.53 Repair of the anulus fibrosus $w /$ graft or prosthesis

80.54 Other \& unspecified repair of the anulus fibrosus

0.59 Other destruction of intervertebral disc

81.00 Spinal fusion, not otherwise specified

81.01 Atlas-axis spinal fusion

81.02 Other cervical fusion, anterior technique

81.03 Other cervical fusion, posterior technique

81.04 Dorsal \& dorsolumbar fusion, anterior technique

11.05 Dorsal \& dorsolumbar fusion, posterior technique

81.06 Lumbar \& lumbosacral fusion, anterior technique

81.08 Lumbar \& lumbosacral fusion, posterior technique

81.30 Refusion of spine, not otherwise specified

1.31 Refusion of atlas-axis spine

1.32 Refusion of other cervical spine, anterior technique

81.33 Refusion of other cervical spine, posterior technique

81.34 Refusion of dorsal \& dorsolumbar spine, anterior technique

81.35 Refusion of dorsal \& dorsolumbar spine, posterior technique

81.36 Refusion of lumbar \& lumbosacral spine, anterior technique

81.39 Refusion of spine, not elsewhere classified

84.51 Insertion of interbody spinal fusion device

of other spinal devices

Insertion of spinal disc prosthesis, not otherwise specified

65 Insertion of total spinal disc prosthesis, lumbosacra cervical 
» CONTINUED FROM PAGE 1580

TABLE 1. Cranial and spine operation codes

\begin{tabular}{cc}
\hline \multicolumn{1}{c}{ Code } & Description \\
\hline Spine (continued) \\
\hline 84.67 & $\begin{array}{c}\text { Revision or replacement of artificial spinal disc prosthesis, } \\
\text { thoracic }\end{array}$ \\
\hline 84.68 & $\begin{array}{c}\text { Revision or replacement of artificial spinal disc prosthesis, } \\
\text { lumbosacral }\end{array}$ \\
\hline 84.69 & $\begin{array}{c}\text { Revision or replacement of artificial spinal disc prosthesis, } \\
\text { not otherwise specified }\end{array}$ \\
\hline 84.80 & $\begin{array}{l}\text { Insertion or replacement of interspinous process device(s) } \\
84.81\end{array}$ \\
\hline 84.82 & $\begin{array}{l}\text { Revision of interspinous process device(s) } \\
\text { zation device(s) }\end{array}$ \\
\hline 84.83 & Revision of pedicle-based dynamic stabilization device(s) \\
\hline 84.84 & Insertion or replacement of facet replacement device(s) \\
\hline 84.85 & Revision of facet replacement device(s) \\
\hline
\end{tabular}

to a short-term hospital, skilled nursing facility, intermediate care facility, home health care, another nursing or rehabilitation facility, or death.

\section{Controlling for Covariates}

Multiple factors are known to influence neurosurgical outcomes, many of which are coded in the NIS. All models controlled for the effects of insurance status, race, sex, age, hospital teaching status, urban versus rural hospital location, whether the operation was completed in an elective setting, and year in which the procedure was performed. Insurance status was separated into 3 groups: patients with private insurance or Medicare, patients with Medicaid, and uninsured patients. Patient comorbidities were assessed using a calculated Charlson Comorbidity Index as defined by Romano et al. ${ }^{17} \mathrm{~A}$ binary variable specifying whether patients were admitted from a long-term health care facility was also used.

\section{Replications of Results}

A replication of cranial specialization results was performed using a cohort of patients undergoing surgical clip occlusion of an intracranial aneurysm (ICD-9-CM code 39.51). An additional replication of spinal specialization results was performed using a cohort of patients undergoing spinal decompression surgery (code 03.09).

\section{Statistical Analysis}

Statistical analysis was performed using commercially available software (STATA/SE 11.2; StataCorp LLC). Multivariate analyses were performed using logistic regression models, adjusting for surgeon spinal or cranial volume, surgeon specialization (that is, percent of spinal or cranial volume relative to total volume), patient insurance status, race, Charlson Comorbidity Index, age, hospital teaching status, hospital urbanicity, year, and adverse admission status. All tests were 2 -sided and statistical significance was established at $\mathrm{p}<0.05$.

\section{Results \\ Data Set}

A total of 231,875 patients were identified as having received an instrumented spine surgery of either the lumbar or cervical spine and were included in the spinal analysis cohort. A total of 46,029 patients were identified as having received an operation for parenchymal excision of a mass lesion or a lobectomy and were included in the cranial analysis cohort. Patient demographic data for both cohorts are provided in Table 2.

There were 4304 neurosurgeon-years with a total volume of $\geq 50$ cases identified as having completed at least 1 operation in the cranial analysis cohort. Among these surgeons, the median total volume of cranial and spinal cases identified in the NIS in a given year was 114 cases (interquartile range [IQR] 80-161 cases) (Table 3). The median number of cranial cases performed by these surgeons in a given year was 29 (IQR 17-49 cases). The median degree of cranial specialization (that is, the percentage of total cases that were cranial in nature) was $25 \%$ (IQR $14 \%-$ $43 \%$ ) in a given year for neurosurgeons operating in the cranial analysis cohort.

There were 4656 neurosurgeon-years with a total volume of $\geq 50$ cases identified as having completed at least 1 operation in the spinal analysis cohort. The median total volume of spinal and cranial cases performed by neurosurgeons in the spinal analysis cohort in a given year in the NIS was 114 cases (IQR 79-162 cases) (Table 3 ). Among neurosurgeons operating in the spinal analysis cohort, the median number of spinal cases performed in a given year was 81 (IQR 52-130 cases). The median degree of spinal specialization (that is, the percentage of total cases dedicated to operations of the spine) was $80 \%$ (IQR 63\%-90\%) for neurosurgeons operating in the spinal analysis cohort.

\section{Specialization and Mortality Patterns Among Neurosurgeons Performing Cranial and Spinal Neurosurgery}

A series of histograms display the distribution of neurosurgeon specialization, total cranial and spinal case volume, and total cranial and spinal patient mortality rates by surgeon specialization. Figure $1 \mathrm{~A}$ reveals that most neurosurgeons concentrate their practice in spinal neurosurgery. Approximately one-half (49.9\%) of all neurosurgeons devoted $>75 \%$ of their practice to spine surgery, and $79.8 \%$ of all neurosurgeons devoted $>50 \%$ of their practice to spinal neurosurgery. In contrast, only $10.2 \%$ of all neurosurgeons devoted $>75 \%$ of their practice to cranial surgery, and $20.2 \%$ of neurosurgeons devoted $>50 \%$ of their practice to cranial surgery.

\section{Results When Operating Within Specialty}

When operating in their majority specialized domain, highly specialized surgeons had a lower than expected rate of mortality. Highly specialized cranial surgeons operated on $24.5 \%$ of all cranial surgery patients (Fig. 1B), accounting for $15.4 \%$ of all cranial surgery deaths (Fig. 1C), and had lower patient mortality rates per surgeon on cranial operations than less specialized surgeons (Fig. 1D). Simi- 
TABLE 2. Characteristics from the NIS of patients undergoing cranial and spine procedures (1998-2009)

\begin{tabular}{|c|c|c|c|c|}
\hline Variable & Available Cranial Data & Cranial & Available Spine Data & Spine \\
\hline Age in yrs & $46,029(100.0)$ & $48.5(52.0)^{*}$ & $231,875(100.0)$ & $52.6(52.0)^{*}$ \\
\hline Race/ethnicity & \multirow[t]{7}{*}{$38,443(84.0)$} & & $192,937(83.0)$ & \\
\hline White & & $29,960(77.9)$ & & $163,185(84.6)$ \\
\hline Black & & $3520(9.2)$ & & $16,082(8.3)$ \\
\hline Hispanic & & $3138(8.2)$ & & $8464(4.4)$ \\
\hline Asian or Pacific Islander & & $481(1.3)$ & & $1202(0.6)$ \\
\hline Native American & & $185(0.5)$ & & $620(0.3)$ \\
\hline Other & & $1159(3.0)$ & & $3384(1.8)$ \\
\hline Sex & \multirow[t]{3}{*}{$46,024(100.0)$} & & $231,850(100.0)$ & \\
\hline Male & & $24,173(52.5)$ & & $111,304(48.0)$ \\
\hline Female & & $21,851(47.5)$ & & $120,546(52.0)$ \\
\hline Calendar year & \multirow[t]{13}{*}{$46,029(100.0)$} & & $231,875(100.0)$ & \\
\hline 1998 & & $2969(6.5)$ & & $10,889(4.7)$ \\
\hline 1999 & & $3482(7.6)$ & & $14,563(6.3)$ \\
\hline 2000 & & $3663(7.9)$ & & $16,327(7.0)$ \\
\hline 2001 & & $3676(8.0)$ & & $15,358(6.6)$ \\
\hline 2002 & & $3800(8.3)$ & & $17,367(7.5)$ \\
\hline 2003 & & $4641(10.1)$ & & $23,709(10.2)$ \\
\hline 2004 & & $4178(9.1)$ & & $21,088(9.1)$ \\
\hline 2005 & & $4003(8.7)$ & & $20,665(8.9)$ \\
\hline 2006 & & $3370(7.3)$ & & $21,711(9.4)$ \\
\hline 2007 & & $4022(8.7)$ & & $20,953(9.0)$ \\
\hline 2008 & & $4461(9.7)$ & & $26,635(11.5)$ \\
\hline 2009 & & $3764(8.2)$ & & $22,610(9.8)$ \\
\hline Charlson Comorbidity Index & $46,029(100.0)$ & $2.64(2.0)^{*}$ & $231,875(100.0)$ & $0.41(0.0)^{*}$ \\
\hline Expected primary payer & \multirow[t]{4}{*}{$44,066(96.0)$} & & $203,400(88.0)$ & \\
\hline Medicare or private insurance & & $36,684(83.3)$ & & $186,135(91.5)$ \\
\hline Medicaid & & $5462(12.4)$ & & $12,865(6.3)$ \\
\hline Uninsured & & $1920(4.4)$ & & $4400(2.2)$ \\
\hline Hospital teaching status & \multirow[t]{3}{*}{$45,882(100.0)$} & & $230,522(99.0)$ & \\
\hline Teaching & & $36,335(79.2)$ & & $133,821(58.1)$ \\
\hline Nonteaching & & $9547(20.8)$ & & $96,701(42.0)$ \\
\hline Hospital location & \multirow[t]{3}{*}{$46,029(100.0)$} & & $231,875(100.0)$ & \\
\hline Urban & & $44,515(96.7)$ & & $222,442(95.9)$ \\
\hline Rural & & $1514(3.3)$ & & $9433(4.1)$ \\
\hline Admission type & \multirow[t]{3}{*}{$32,167(70.0)$} & & $174,429(75.0)$ & \\
\hline Elective & & $17,300(53.8)$ & & $149,475(85.7)$ \\
\hline Nonelective & & $14,867(46.2)$ & & $24,954(14.3)$ \\
\hline Admission source & \multirow[t]{3}{*}{$46,029(100.0)$} & & $231,875(100.0)$ & \\
\hline Routine & & $45,365(98.6)$ & & $230,888(99.6)$ \\
\hline Transfer from another health facility & & $664(1.4)$ & & $987(0.4)$ \\
\hline
\end{tabular}

Data are the number of patients (\%) unless otherwise specified.

* Data are the mean (median).

larly, specialized spinal surgeons performed $74.6 \%$ of all spinal surgeries (Fig. 1E) but accounted for only $57.0 \%$ of all spinal surgery deaths (Fig. 1F) and had lower patient mortality rates per surgeon on spine operations than less specialized neurosurgeons (Fig. 1G).

We noted that increases in surgeon cranial specialization are associated with lower rates of cranial mortality across all levels of surgeon total volume in a stepwise fashion (Fig. 2A). We noted similar trends when investigating adverse discharge disposition in the cranial analysis cohort. Similar trends were observed when investigating mortality rates and adverse discharge disposition in the spinal analysis cohort. Figure 2B-D demonstrates a reverse J-shape curve, in which rates of mortality and adverse discharge 
TABLE 3. Surgeon case mix characteristics among surgeons identified in the spinal and cranial cohorts

\begin{tabular}{cc}
\hline \multicolumn{1}{c}{ Cohort } & Median (IQR) \\
\hline Spine & \\
\hline Surgeon vol of cases, no. & $81(52-130)$ \\
\hline Surgeon \% of total cases & $80(63-90)$ \\
\hline Surgeon total vol of cases, no. & $114(79-162)$ \\
\hline Cranial & $29(17-49)$ \\
\hline Surgeon vol of cases, no. & $25(14-43)$ \\
\hline Surgeon \% of total cases & $114(80-161)$ \\
\hline Surgeon total vol of cases, no.
\end{tabular}

disposition seem to increase slightly for neurosurgeons with the very highest levels of specialization.

\section{Specialization Demonstrates a Dose-Response Type of Relationship With Patient Mortality}

Figure 3 displays mortality rates for each surgeon in a given year. Each neurosurgeon-year is displayed as a point in a scatter plot of overall operative volume (cranial plus spinal cases) versus specialization in the cranial analysis cohort and spinal analysis cohort. Coloring indicates the quintile grouping of mortality for each neurosurgeon-year plotted.

As seen in Fig. 3 (left), neurosurgeons with the highest cranial mortality rates form a cluster in the lower left region of the scatter plot, which represents low volume and low cranial specialization. Higher degrees of cranial specialization, even among surgeons with relatively low volume, are still seen to be associated with significant improvements in mortality rate. Neurosurgeons in the quintile with the lowest patient mortality tended to demonstrate $\geq 50 \%$ cranial specialization across a wide range of total yearly case volumes. The cranial surgery mortality rate and range in each quintile are provided in Table 4.

In Fig. 3 (right), we note similar trends in the spinal analysis cohort. Neurosurgeons with the highest spinal surgery mortality rates cluster in the lower left region of the scatter plot, which represents low volume and low specialization. Surgeons with the lowest spinal surgery mortality rates cluster in the upper right corner of the plot,

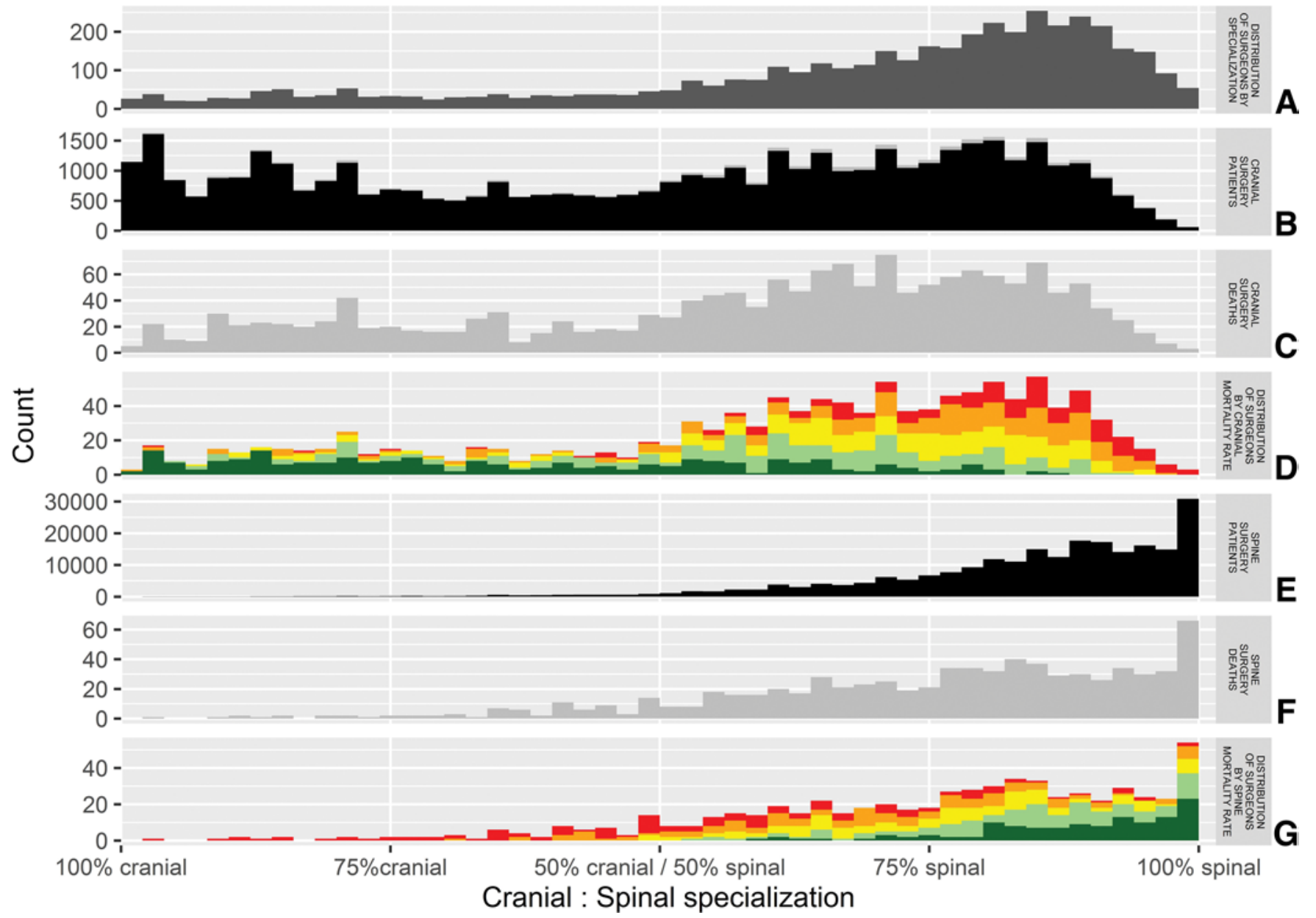

FIG. 1. Histograms showing the number of neurosurgeons (A) as a function of neurosurgeon specialization ( $x$ axis), indicating that most neurosurgeons are specialized in spinal surgery. B-D: Histograms showing the number of cranial surgery patients (B) and deaths $(C)$, and the mortality rate per surgeon (D). E-G: Histograms showing the number of spinal surgery patients $(E)$ and deaths $(F)$, and the mortality rate per surgeon $(G)$, respectively. In panels $D$ and $G$, colors represent mortality rate ranges from lower (green) to higher (red). 

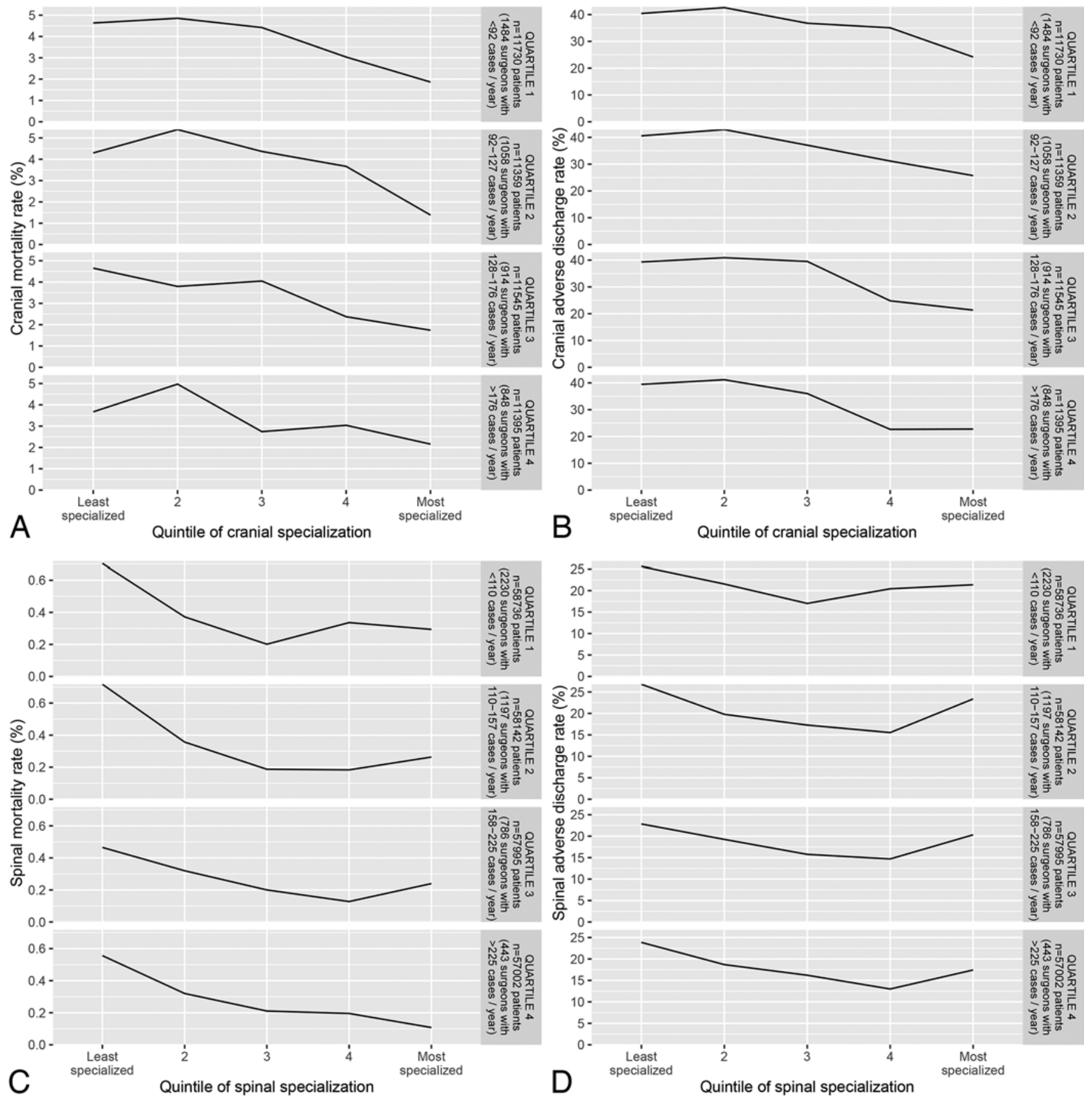

FIG. 2. A and B: Mortality rate and adverse discharge disposition for cranial surgeries (y axis) shown for 4 groups of surgeons categorized by total surgical volume (1st quartile < 92 cases per year, 2nd quartile 92-127 cases per year, 3rd quartile 128-176 cases per year, 4th quartile $>176$ cases per year). The $x$ axis shows neurosurgeon specialization subcategorized into quintiles according to the percentage of overall practice that is cranial surgery. C and D: Mortality rate and adverse discharge disposition for spinal surgeries (y axis) shown for 4 groups of surgeons categorized by total surgical volume (1st quartile < 110 cases per year, 2nd quartile 110-157 cases per year, 3rd quartile 158-225 cases per year, 4 th quartile $>225$ cases per year). The $x$ axis shows neurosurgeon specialization subcategorized into quintiles according to the percentage of overall practice that is spinal surgery.

which represents greater volume and specialization. Overall operative volume seems to have a stronger association with improvements in mortality rate in the spinal analysis cohort relative to the cranial analysis cohort. In Table 4, the mortality rate and range in each quintile for spinal surgery are shown.

\section{Magnitude of the Specialization Effect in Cranial Surgery}

When patient mortality was investigated in a logistic regression model in the cranial analysis cohort (Table 5), surgeon specialization and surgeon total volume were significantly associated with patient mortality. Using surgeon total volume as a continuous variable, each additional sur- 

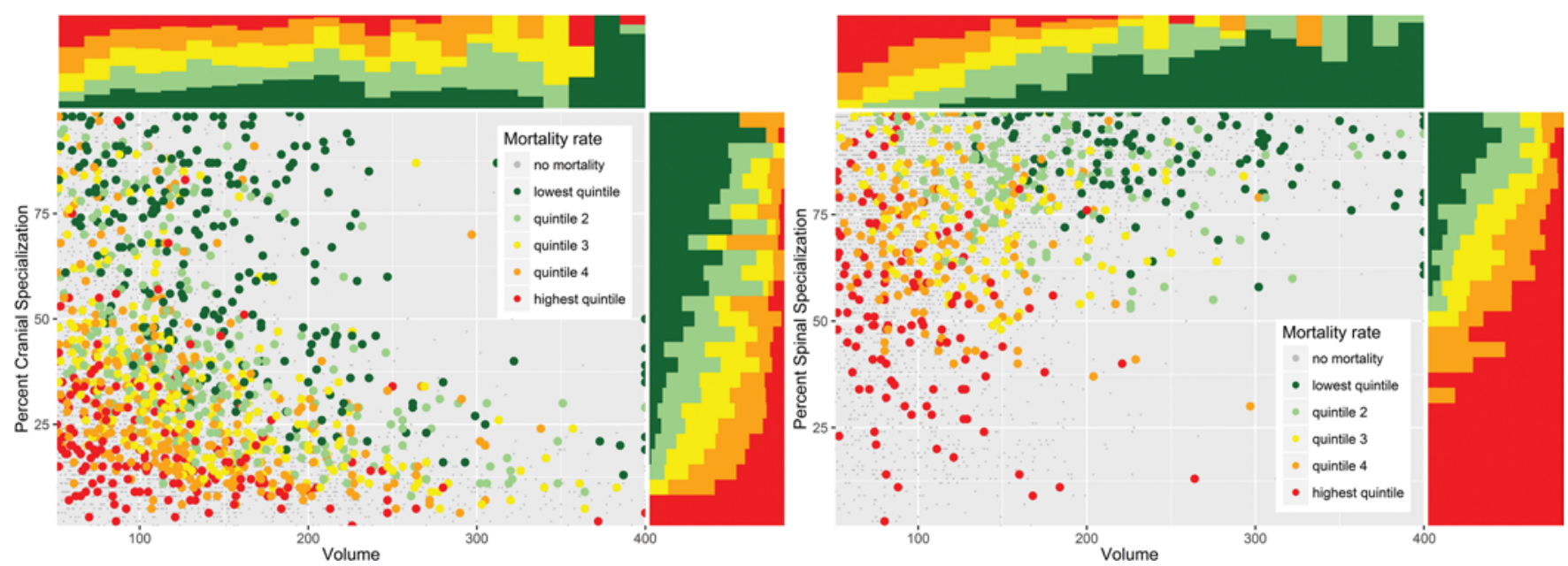

FIG. 3. Scatter plots displaying mortality rates (green represents lower, red represents higher) as a function of case volume ( $\mathrm{x}$ axis) and percent spinal or cranial surgery specialization (y axis) among surgeons with at least 1 patient fatality. The cranial data (left) illustrate a clear trend toward lower mortality rates with increasing specialization; however a weaker trend toward increased case volume is noted. In the spine data (right), a clear trend toward lower mortality rates can be seen with both increasing volume and increasing specialization. Histograms summarizing mortality rates as a function of case volume (above the scatter plots) and specialization (to the right of scatter plots) are provided as summary statistics.

gical case was associated with a $0.0010(95 \%$ CI $0.0002-$ $0.0019)$ reduction in the log odds of mortality. With each percentage-point increase in surgeon cranial specialization, there was an associated 0.0060 reduction in the $\log$ odds of patient mortality (95\% CI 0.0034-0.0086). A marginal effects analysis demonstrated that the magnitude of this association was such that there was a $15 \%$ reduction in the predicted probability of mortality as neurosurgeon cranial specialization increased from 14\% (25th percentile) to $43 \%$ (75th percentile).

When multivariate analysis was performed, both surgeon total volume and surgeon specialization were associated with significant reductions in the predicted probability of adverse discharge disposition for patients in the cranial analysis cohort. Each additional case in a surgeon's total volume was associated with a 0.0009 reduction in the log odds of adverse discharge (95\% CI 0.0006-0.0013). Likewise, each percentage-point increase in surgeon cra- nial specialization was associated with a 0.0042 reduction in the log odds of patient adverse discharge disposition (95\% CI 0.0032-0.0052). When comparing surgeons operating at the 75th percentile of surgeon cranial specialization with those operating at the 25 th percentile, we observed a $6.1 \%$ difference in the predicted probability of patient adverse discharge disposition.

\section{Magnitude of the Specialization Effect in Spinal Surgery}

In the spinal analysis cohort, multivariate analysis revealed that increases in surgeon total volume were associated with a downward trend in the predicted probability of patient mortality (Table 6). However, this trend was not statistically significant. The degree of spinal specialization was associated with a significant reduction in the predicted probability of patient mortality after risk adjustment for available patient and hospital information. Each percent-

TABLE 4. Characteristics of surgeon mortality quintiles for cranial and spinal analysis cohorts

\begin{tabular}{cccccc}
\hline Quintile & $\begin{array}{c}\text { Patient Mortality Rate } \\
(\%) \text { by Surgeon, Range }\end{array}$ & $\begin{array}{c}\text { No. of Surgeon- } \\
\text { Yrs in Quintile }\end{array}$ & $\begin{array}{c}\text { No. of Patients } \\
\text { in Quintile }\end{array}$ & $\begin{array}{c}\text { No. of Deaths } \\
\text { in Quintile }\end{array}$ & $\begin{array}{c}\text { Overall Patient Mortality } \\
\text { Rate (\%) in Quintile }\end{array}$ \\
\hline Cranial & & & & & \\
\hline 1 & $0.69-5.58$ & 253 & 9009 & 299 & 3.32 \\
\hline 2 & $5.58-9.09$ & 255 & 4157 & 312 & 7.51 \\
\hline 3 & $9.09-14.3$ & 270 & 2939 & 350 & 11.91 \\
\hline 4 & $14.3-25.0$ & 254 & 2041 & 398 & 19.50 \\
\hline 5 & $25.0-100$ & 179 & 700 & 276 & 39.43 \\
\hline Spinal & & & & & \\
\hline 1 & $0.26-1.03$ & 126 & 19,224 & 133 & 0.69 \\
\hline 2 & $1.03-1.72$ & 128 & 10,475 & 140 & 1.34 \\
\hline 3 & $1.72-2.60$ & 122 & 6616 & 139 & 2.10 \\
\hline 4 & $2.60-4.25$ & 125 & 4932 & 162 & 3.28 \\
\hline 5 & $4.25-100$ & 126 & 2581 & 170 & 6.59 \\
\hline
\end{tabular}


TABLE 5. Results of multivariate logistic regression analysis displaying log odds of death and adverse discharge for patients undergoing cranial surgery

\begin{tabular}{|c|c|c|c|c|}
\hline \multirow[b]{2}{*}{ Variable } & \multicolumn{2}{|c|}{ Adjusted Mortality, $n=36,681$} & \multicolumn{2}{|c|}{ Adjusted Adverse Discharge, $n=36,640$} \\
\hline & Log Odds (95\% Cl) & $p$ Value & Log Odds (95\% Cl) & p Value \\
\hline \multicolumn{5}{|l|}{ Surgeon case mix } \\
\hline Total vol & $-0.0010(-0.0019$ to -0.0002$)$ & 0.015 & $-0.0009(-0.0013$ to -0.0006$)$ & $<0.001$ \\
\hline$\%$ of total cases, cranial & $-0.0060(-0.0086$ to -0.0034$)$ & $<0.001$ & $-0.0042(-0.0052$ to -0.0032$)$ & $<0.001$ \\
\hline
\end{tabular}

age-point increase in the proportion of cases dedicated to spinal practice was significantly associated with a 0.0122 reduction in the log odds of mortality (95\% CI 0.0074 0.0170). When marginal effects analysis was performed, a $26.8 \%$ reduction in the predicted probability of patient mortality was observed as neurosurgeon spinal specialization increased from 63\% (25th percentile) to 90\% (75th percentile).

Multivariate analysis of adverse discharge disposition in the spinal analysis cohort demonstrated a significant association with surgeon total volume and specialization. Each additional neurosurgical case was associated with a 0.0007 reduction in the log odds of adverse discharge disposition (95\% CI 0.0006-0.0009). Each percentage-point increase in spinal specialization was associated with a 0.0058 reduction in the log odds of adverse discharge disposition (95\% CI 0.0049-0.0067). This resulted in a $9.6 \%$ reduction in the predicted probability of adverse discharge disposition between surgeons operating at the 75th percentile and the 25th percentile for spinal specialization.

\section{Sensitivity Analysis}

A sensitivity analysis was performed to investigate whether the results described above were reproducible in the population of patients undergoing surgical clip occlusion of an intracranial aneurysm (ICD-9-CM code 39.51) in the NIS data set. On multivariate analysis, neurosurgeon cranial specialization was again associated with a significant and independent reduction in patient mortality ( $\log$ odds $-0.006,95 \%$ CI -0.009 to -0.002 ), such that the predicted probability of mortality decreased by $14.4 \%$ as neurosurgeon specialization increased from $14 \%$ (25th percentile) to $43 \%$ (75th percentile) after controlling for other variables. On multivariable analysis fitted for adverse discharge disposition, increasing neurosurgeon cranial specialization was associated with a small but significant reduction in the log odds of adverse discharge disposition (log odds $-0.005,95 \% \mathrm{CI}-0.007$ to -0.003 ). This translated to a $5.8 \%$ reduction in the predicted probability of adverse discharge between the 25 th and 75 th percentiles of neurosurgeon cranial specialization.

An additional sensitivity analysis was performed to investigate whether similar trends with regard to surgeon specialization could be observed in the population of patients undergoing spinal decompression surgery (ICD-9CM code 03.09). On multivariate analysis, neurosurgeon specialization was associated with a reduction in both mortality (log odds $-0.017,95 \%$ CI -0.021 to -0.012 ) and adverse discharge disposition (log odds $-0.006,95 \% \mathrm{CI}$ -0.007 to -0.005 ). There was a $5.2 \%$ associated reduction in the predicted probability of mortality as neurosurgeon spinal specialization increased from $63 \%$ (25th percentile) to $90 \%$ (75th percentile). Similarly, there was a $9.9 \%$ associated reduction in the predicted probability of adverse discharge disposition as neurosurgeon specialization increased from the 25 th to the 75 th percentile.

\section{Discussion}

This study investigated the association between spinal and cranial specialization and patient outcomes among neurological surgeons included in the NIS database. Our data suggest that both the volume of cases and the subspecialization of that volume are associated with improved patient outcomes. Because neurosurgeons are trained in both spinal and cranial neurosurgical procedures and they frequently develop a practice profile that involves performing both types of procedures, and because the degree of specialization can be modified for individual surgeons in a group, it is of interest to study the effect of practice specialization on patient outcomes to inform decision making by individual surgeons or groups of surgeons regarding the impact of their specialization choices.

When patient mortality was investigated using a logistic regression model, increased surgeon specialization was significantly associated with reductions in the predicted probability of death in both the cranial and spinal analysis cohorts. Total volume was also significantly associated

TABLE 6. Results of multivariate logistic regression analysis displaying log odds of death and adverse discharge for patients undergoing spine surgery

\begin{tabular}{|c|c|c|c|c|}
\hline \multirow[b]{2}{*}{ Variable } & \multicolumn{2}{|c|}{ Adjusted Mortality, $n=168,856$} & \multicolumn{2}{|c|}{ Adjusted Adverse Discharge, $n=168,708$} \\
\hline & Log Odds (95\% Cl) & $p$ Value & Log Odds (95\% Cl) & $\mathrm{p}$ Value \\
\hline \multicolumn{5}{|l|}{ Surgeon case mix } \\
\hline Total vol & $-0.0010(-0.0021$ to 0.0001$)$ & 0.062 & $-0.0007(-0.0009$ to -0.0006$)$ & $<0.001$ \\
\hline$\%$ of total cases, spine & $-0.0122(-0.0170$ to -0.0074$)$ & $<0.001$ & $-0.0058(-0.0067$ to -0.0049$)$ & $<0.001$ \\
\hline
\end{tabular}


with a reduction in patient mortality in the cranial analysis cohort. Similar trends were elucidated for patient adverse discharge disposition. In general, the trends of improved mortality and discharge outcomes with improved specialization increased monotonically, as shown in Fig. 2A-D. However, we did note a slight uptick in mortality rate and adverse morbidity among the most highly specialized spine surgeons. This finding may be explained if highly specialized spine surgeons take on significantly more complex or difficult operative cases.

The findings in this study suggest that both higher volume and subspecialization are associated with a beneficial impact on patient mortality and morbidity. The finding that surgeon case volume correlates with outcome is not unique and has been extensively documented as the volume-outcome relationship in the medical and surgical literature. ${ }^{5,20}$ This study adds an additional dimension to the traditional volume-outcome relationship: surgeon specialization. The distinction between higher volume and specialization is important because it provides neurosurgeons with 2 unique opportunities to improve their outcomes. It is a nontrivial challenge to simply increase the quantity of cases in one's practice. However, it may be more feasible to organize with partners and establish a division of labor such that each individual surgeon is maintaining a more specialized practice.

This analysis raises the question of whether tipping points of specialization exist such that above a given level of specialization, the outcomes in the area of major specialization are so good and outcomes in the area of minor specialization are so poor that there may be a significant benefit for the surgeon to become fully specialized in 1 clinical domain. This would probably occur near the extremes of either spinal or cranial specialization. Because of this phenomenon, in some situations, it may be prudent for a surgeon to organize their practice to be more fully specialized in 1 particular domain of neurosurgery if they are working in an environment that is permissive for such restructuring.

We envision that the data presented herein could serve as a basis for modeling how neurosurgical groups could achieve an optimal specialization within their groups to ensure the best overall outcomes for the population they serve. For example, a 3-surgeon group may require 3 surgeons with the typical practice mix of $80 \%$ spine and $20 \%$ cranial to meet the demands of call coverage, whereas an 8-surgeon group may be best served with 2 majority cranial practitioners and 6 majority spine practitioners to improve clinical outcomes. Carefully designed future studies may further illustrate the benefits of such changes.

These results may also impact the organization of practice in academic groups, where teaching and/or research may comprise a significant portion of a given faculty member's efforts. These data suggest that lower-volume, but highly specialized, cranial or spinal surgeons can achieve excellent outcomes. Thus, a faculty member who elects to devote significant professional effort to research or teaching may retain expert-level outcomes through specialization.

This study is strengthened by the fact that the NIS is a large, generalizable data set that is representative of the general US population. The NIS data set also allowed us to test a hypothesis that would be difficult to explore using alternate methodologies. To date, the NIS has been used in numerous studies to investigate the association between provider volume and patient outcome. ${ }^{1,9}$ Because specialization was defined as the ratio of spinal or cranial volume relative to total volume, which is readily available for calculation in the NIS, the NIS is a valid data source for this study. This study also investigated the role of subspecialization in the 2 major clinical divisions within neurosurgery: spinal and cranial surgery. Therefore, the results are particularly germane to multiple stakeholders within the neurosurgical community.

This study is associated with all of the attendant weaknesses attributed to administrative data sets. Errors in administrative coding exist. However, it is unlikely that such errors are biased toward specialized versus unspecialized neurosurgeons in this study, and therefore these errors are unlikely to have an impact on our results. Highly specific clinical details and imaging are notably absent from the data used for this study. As a result, risk adjustment is imperfect, and it is difficult to fully assess other patient or operative characteristics that may be relevant. In addition, the ICD-9-CM codes used for patient selection are specific, and the results of this study may not be generalizable to other neurosurgical operations.

Moreover, for neurosurgeons who operate at $>1$ facility, this study may not capture the entirety of their practice and the total volumes presented may underestimate true volumes. The NIS also does not provide information regarding whether a neurosurgeon has completed fellowship training. Others may prefer to define specialization according to whether fellowship training was completed, and this study cannot provide insight in this regard.

Given limitations in the granularity of data provided in the NIS, future studies will be needed to determine in more detail the impact of specific subspecialization (e.g., tumor, cerebrovascular, trauma) on patient outcomes. Further studies that use data sources with longer follow-up and functional outcome measures (e.g., long-term neurological sequelae or patient disability indices at 1 year) will also be needed.

There is a trend toward centralized medical and surgical care across the United States. Large, centralized practices may organize as a group of generalists or maintain a division of labor according to surgeon subspecialization. This study suggests that subspecialized neurosurgeons are associated with improvements in patient morbidity and mortality when operating in their realm of specialization. This study may, therefore, argue in favor of earlier subspecialty training.

\section{Conclusions}

Taken as a whole, our data suggest that the best outcomes are seen among surgeons who perform surgeries within their realm of subspecialization. Our data further suggest that even surgeons with an overall lower-volume practice may achieve excellent outcomes by specialization. These results also confirm those of prior studies, which have shown that high-volume surgeons have lower rates of inpatient mortality and adverse discharge disposition for both spinal and cranial surgery. These findings support the 
current trend toward subspecialization seen in our contemporary health care system.

\section{References}

1. Al-Qurayshi Z, Robins R, Hauch A, Randolph GW, Kandil E: Association of surgeon volume with outcomes and cost savings following thyroidectomy: a national forecast. JAMA Otolaryngol Head Neck Surg 142:32-39, 2016

2. Albright AL, Sposto R, Holmes E, Zeltzer PM, Finlay JL, Wisoff JH, et al: Correlation of neurosurgical subspecialization with outcomes in children with malignant brain tumors. Neurosurgery 47:879-887, 2000

3. Birkmeyer JD, Stukel TA, Siewers AE, Goodney PP, Wennberg DE, Lucas FL: Surgeon volume and operative mortality in the United States. N Engl J Med 349:2117-2127, 2003

4. Cassel CK, Reuben DB: Specialization, subspecialization, and subsubspecialization in internal medicine. N Engl J Med 364:1169-1173, 2011

5. Chowdhury MM, Dagash H, Pierro A: A systematic review of the impact of volume of surgery and specialization on patient outcome. Br J Surg 94:145-161, 2007

6. Couldwell WT, Rovit RL: Rethinking neurosurgical subspecialization. Surg Neurol 58:359-370, 2002

7. Cowan JA Jr, Dimick JB, Leveque JC, Thompson BG, Upchurch GR Jr, Hoff JT: The impact of provider volume on mortality after intracranial tumor resection. Neurosurgery 52:48-54, 2003

8. Curry WT Jr, Carter BS, Barker FG II: Racial, ethnic, and socioeconomic disparities in patient outcomes after craniotomy for tumor in adult patients in the United States, 19882004. Neurosurgery 66:427-438, 2010

9. De la Garza-Ramos R, Abt NB, Kerezoudis P, Krauss W, Bydon M: Provider volume and short-term outcomes following surgery for spinal metastases. J Clin Neurosci 24:43-46, 2016

10. Dimick JB, Cowan JA Jr, Stanley JC, Henke PK, Pronovost PJ, Upchurch GR Jr: Surgeon specialty and provider volumes are related to outcome of intact abdominal aortic aneurysm repair in the United States. J Vasc Surg 38:739-744, 2003

11. Durham SR, Shipman SA: A 15 -year review of pediatric neurosurgical fellowships: implications for the pediatric neurosurgical workforce. J Neurosurg Pediatr 1:429-432, 2008

12. Eppsteiner RW, Csikesz NG, McPhee JT, Tseng JF, Shah SA: Surgeon volume impacts hospital mortality for pancreatic resection. Ann Surg 249:635-640, 2009

13. Golledge J, Wiggins JE, Callam MJ: Effect of surgical subspecialization on breast cancer outcome. Br J Surg 87:14201425,2000
14. Lapar DJ, Bhamidipati CM, Walters DM, Stukenborg GJ, Lau CL, Kron IL, et al: Primary payer status affects outcomes for cardiac valve operations. J Am Coll Surg 212:759-767, 2011

15. Mateo Vallejo F: General surgery: present and future. Int J Surg 10:176-177, 2012

16. McCutcheon BA, Talamini MA, Chang DC, Rose JA, Bandyk DF, Wilson SE: The comparative effectiveness of surgeons over interventionalists in endovascular repairs of abdominal aortic aneurysm. Ann Surg 258:476-482, 2013

17. Romano PS, Roos LL, Jollis JG: Adapting a clinical comorbidity index for use with ICD-9-CM administrative data: differing perspectives. J Clin Epidemiol 46:1075-1090, 1993

18. Scott RM: The fellowship in pediatric neurosurgery. Pediatr Neurosurg 17:281-283, 1991-1992

19. Smith ER, Butler WE, Barker FG II: Craniotomy for resection of pediatric brain tumors in the United States, 1988 to 2000: effects of provider caseloads and progressive centralization and specialization of care. Neurosurgery 54:553565,2004

20. von Meyenfeldt EM, Gooiker GA, van Gijn W, Post PN, van de Velde CJH, Tollenaar RAEM, et al: The relationship between volume or surgeon specialty and outcome in the surgical treatment of lung cancer: a systematic review and meta-analysis. J Thorac Oncol 7:1170-1178, 2012

\section{Disclosures}

The authors report no conflict of interest concerning the materials or methods used in this study or the findings specified in this paper.

\section{Author Contributions}

Conception and design: Carter, McCutcheon, Hirshman, Chen, Chang. Acquisition of data: Carter, McCutcheon, Hirshman, Chen, Chang. Analysis and interpretation of data: McCutcheon, Hirshman, Heffner, Marcus, Cole. Drafting the article: McCutcheon, Hirshman, Gabel, Heffner. Critically revising the article: all authors. Reviewed submitted version of manuscript: Carter, Hirshman, Gabel, Heffner, Marcus, Cole, Chen, Chang. Approved the final version of the manuscript on behalf of all authors: Carter. Statistical analysis: McCutcheon, Hirshman. Administrative/ technical/material support: Carter, McCutcheon, Heffner. Study supervision: Carter, McCutcheon.

\section{Correspondence}

Bob S. Carter, Massachusetts General Hospital, 55 Fruit St., Boston, MA 02114. email: bcarter@mgh.harvard.edu. 